\title{
MFGE8, ALB, APOB, APOE, SAA1, A2M, and C3 as Novel Biomarkers for Stress Cardiomyopathy
}

\author{
Xiao-Yu Pan $\mathbb{D}^{1,2}$ and Zai-Wei Zhang $\mathbb{D}^{2,3}$ \\ ${ }^{1}$ Department of Clinical Medical College, Jining Medical University, Jining, Shandong 272067, China \\ ${ }^{2}$ Department of Cardiology, Jining No. 1 People's Hospital, Jining, Shandong 272011, China \\ ${ }^{3}$ Cardiovascular Research Institute, Jining No.1 People's Hospital, Jining, Shandong 272011, China
}

Correspondence should be addressed to Zai-Wei Zhang; zhzw2001@yeah.net

Received 14 March 2020; Accepted 2 June 2020; Published 1 July 2020

Guest Editor: Annalisa Romani

Copyright (C) 2020 Xiao-Yu Pan and Zai-Wei Zhang. This is an open access article distributed under the Creative Commons Attribution License, which permits unrestricted use, distribution, and reproduction in any medium, provided the original work is properly cited.

\begin{abstract}
Background. Stress cardiomyopathy (SCM) is a transient reversible left ventricular dysfunction that more often occurs in women. Symptoms of SCM patients are similar to those of acute coronary syndrome (ACS), but little is known about biomarkers. The goals of this study were to identify the potentially crucial genes and pathways associated with SCM. Methods. We analyzed microarray datasets GSE95368 derived from the Gene Expression Omnibus (GEO) database. Firstly, identify the differentially expressed genes (DEGs) between SCM patients in normal patients. Then, the DEGs were used for Gene Ontology (GO) and Kyoto Encyclopedia of Genes and Genomes (KEGG) pathway enrichment analysis. Finally, the protein-protein interaction (PPI) network was constructed and Cytoscape was used to find the key genes. Results. In total, 25 DEGs were identified, including 10 upregulated genes and 15 downregulated genes. These DEGs were mainly enriched in ECM-receptor interaction, dilated cardiomyopathy (DCM), human papillomavirus infection, and focal adhesion, whereas in GO function classification, they were mainly enriched in the extracellular region, positive regulation of the multicellular organismal process, establishment of localization, and intracellular vesicle. Conclusion. Seven hub genes contained APOE, MFGE8, ALB, APOB, SAA1, A2M, and C3 identified as hub genes of SCM, which might be used as diagnostic biomarkers or molecular targets for the treatment of SCM.
\end{abstract}

\section{Introduction}

Stress cardiomyopathy, also known as takotsubo cardiomyopathy (TTC), was first reported in Japan in 1990 [1], characterized by transient systolic and diastolic left ventricular (LV) dysfunction with multiple wall dyskinesia, most commonly occurring in postmenopausal women, especially in populations with a recent history of mental or physical stress [2]. The reversibility of cardiac insufficiency is one of the most prominent features of the disease. It is sometimes considered fairly benign, but it has a $4.1 \%$ in-hospital mortality rate, especially in the early stages analogous to acute coronary syndrome (ACS), and is also likely to have cardiogenic shock and fatal arrhythmias [3]. It estimates that 1 to $2 \%$ of suspected ACS patients were eventually diagnosed with stress cardiomyopathy.
The exact pathophysiology of stress cardiomyopathy is unknown and seems to be associated with excess plasma catecholamine, which is caused by stress conditions. Myocardial ischemia appears to play a crucial role in takotsubo syndrome, both in human heart muscle specimens and in experimental models of SCMP. Most cases occur in patients with risk factors for endothelial dysfunction [4].

Most patients with stress-induced cardiomyopathy (>95\%) have electrocardiogram abnormalities that typically show ischemic ST-segment and T-wave changes, but his appearance is most likely to associate the presence of ACS than SCM $[5,6]$. Previous studies have shown that cardiac biomarkers such as cardiac troponin T or I, creatine-kinase myocardial band (CK-MB), and b-type natriuretic peptide (BNP) may play a role in the early clinical recognition of SCM, but the accuracy is low $[7,8]$. In patients with suspected stress 

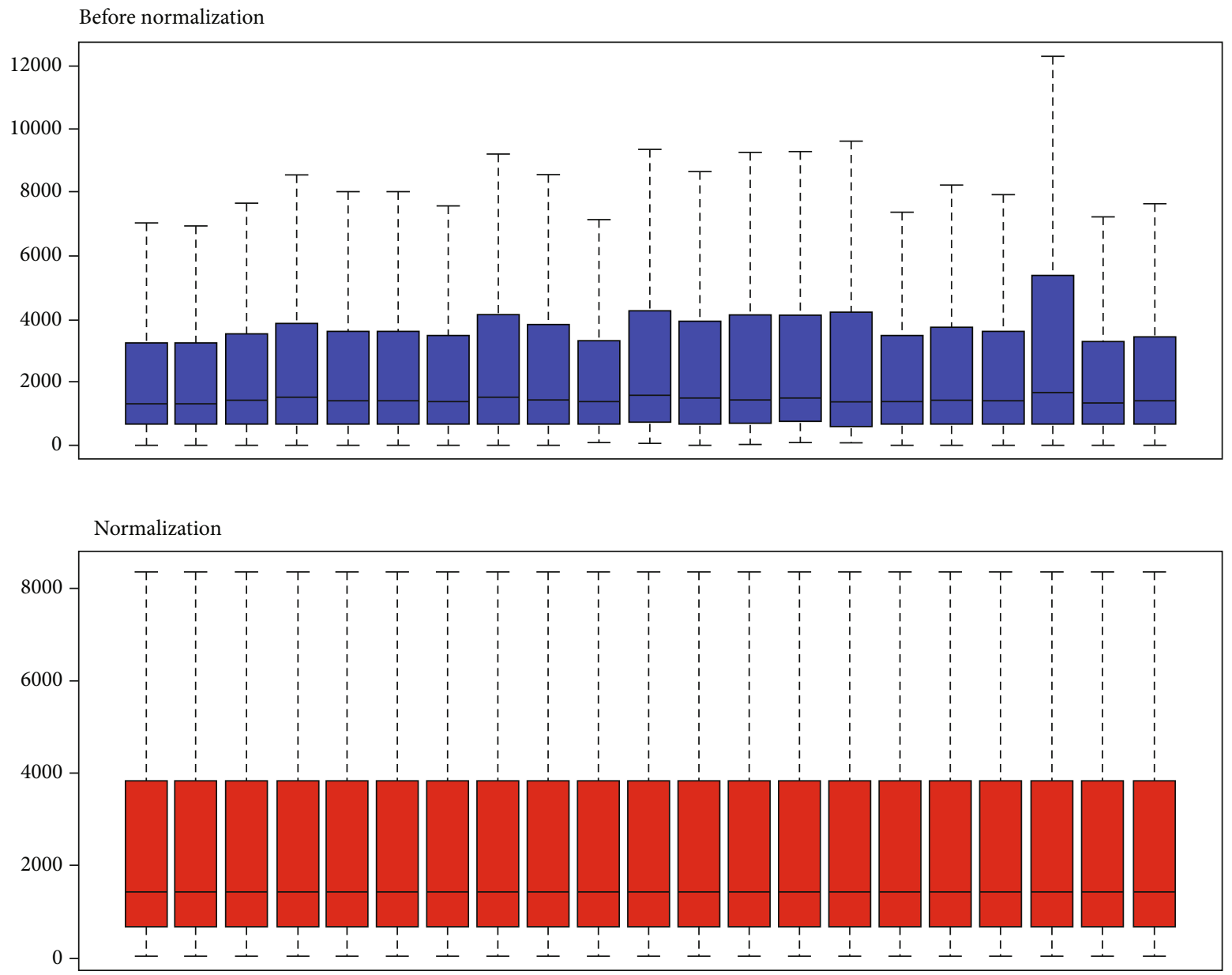

FIGURE 1: Standardization of gene expression. The blue bar represents the data before normalization, and the red bar represents the normalized data.

cardiomyopathy, transthoracic echocardiography with color and tissue doppler is the preferred noninvasive imaging trial [5]. A recent study reports that STE echocardiographic index may be more accurate and unique than traditional echocardiography for the early detection of subtle abnormalities [9]. Because of the reversibility of SCM's condition, it is not possible to determine the diagnosis at the time of presentation, although some clinical features are highly predictive of stressful myocardial disease [10].

In this study, we further revealed biomarkers related to SCM by analyzing gene expression profile (GSE95368) deposited by Yvonne Edwards et al. (2017). Identifying key genes and pathways contributes to a better understanding of the pathophysiological mechanism of disease development, which provides innovative ideas for the diagnosis and treatment of SCM.

\section{Materials and Methods}

2.1. Data Sources. Gene expression data of GSE95368 is available for download from the NCBI Gene Expression Omnibus (GEO; http://www.ncbi.nlm.nih.gov/geo). The expression profiling arrays were generated using the GPL23119 platform (SOMAscan human proteomic $1.3 \mathrm{k}$ assay). A total of 21 serum samples in this database, including 15 from SCM patients and 6 from normal serum samples, were analyzed.

2.2. Data Preprocessing and Identification of DEGs. Genetic matrix files and platform files were downloaded to eliminate errors and make the experimental group comparable between control groups, so the obtained data were standardized. The estimation package is based on the KNN (k-nearest neighbor) algorithm is used to fill the missing values. After this, the probes were converted into gene symbols on the basis of the annotation platform file. If there are multiple probes corresponding to a gene, take the average as the final value. If the probe without gene symbol was removed, the normalized between array function in the limma package is applied to standardize the data. Then, the expression data were log2 transformed and the limma functional package in $\mathrm{R}$ software was used to compare gene expression in SCM and control samples to identify DEGs. The screening criteria for DEGs were $p$ value $<0.05$ and $\mid \log 2$ foldchange $(\mathrm{FC}) \mid \geq 1$.

2.3. GO and KEGG Pathway Enrichment Analysis of DEGs. Database for Annotation, Visualization and Integrated Discovery (DAVID) (https://david.ncifcrf.gov) and KOBAS 
TABLE 1: Screening upregulated and downregulated DEGs.

\begin{tabular}{lc}
\hline DEGs & Gene symbol \\
\hline Upregulated (15) & LTA4H APOB ALB IL36A PRKACA NAMPT XPNPEP1 \\
& ITGA2B ITGB3 CA13 C3 EHMT2 PTPN6 AZU1 A2M MFGE8 \\
Downregulated (10) & GAPDH APOE CRP NPPB PKM2 GDF15 PLAT \\
\hline
\end{tabular}

Abbreviation: DEGs: differentially expressed genes.

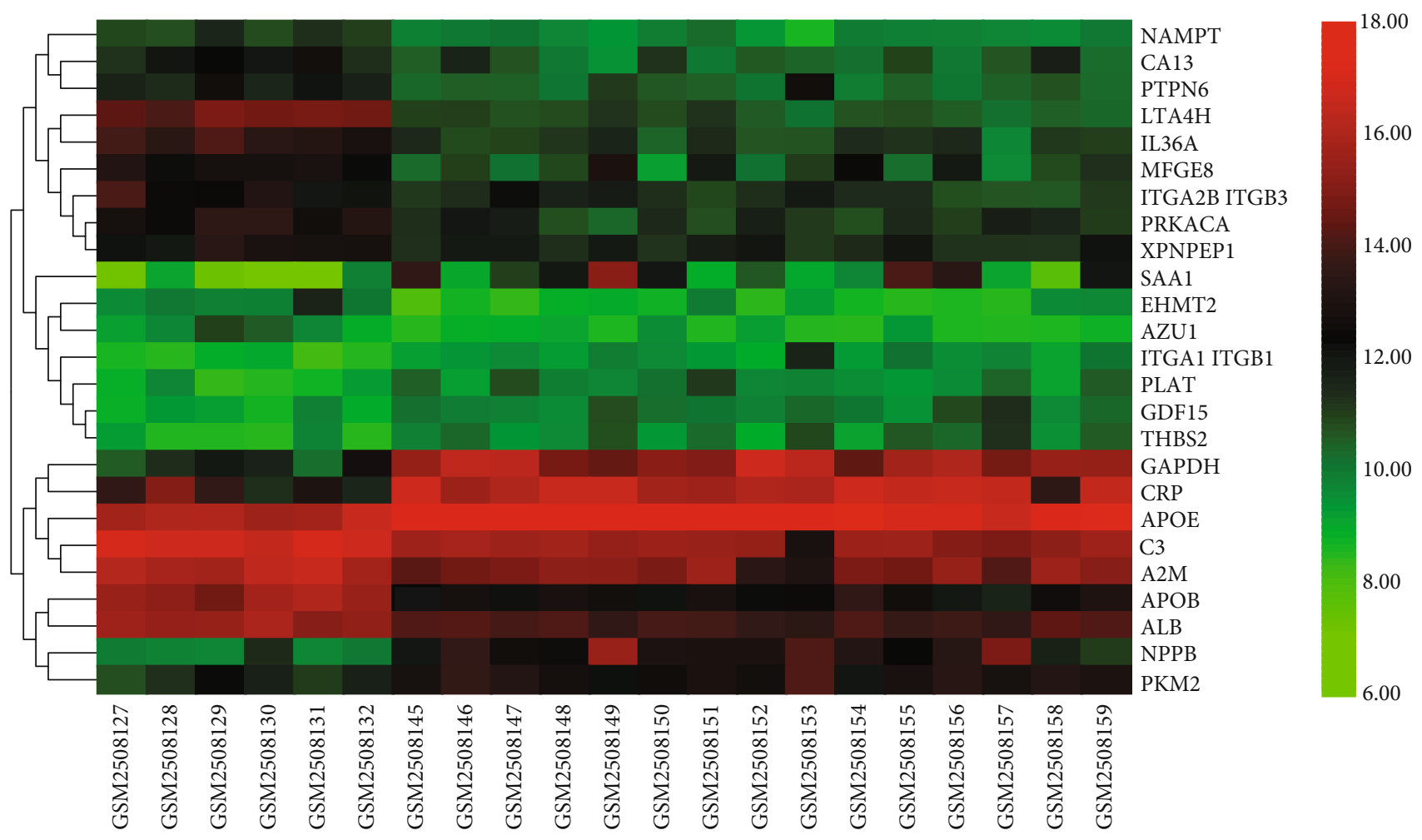

FIgUre 2: Heatmap results of DEGs. Abbreviation: DEGs, differentially expressed genes.

(http://kobas.cbi.pku.edu.cn) were applied for GO annotation and KEGG pathway analysis. Because the KOBAS online tool uses gene ID for data analysis, so we first use DAVID to convert the gene symbol of DEGs into gene ID and then use KOBAS for GO and KEGG enrichment analysis of DEGs. Finally, we use R software to visualize the results, to further understand the potential functions of the identified DEGs.

2.4. PPI Network Analyses. PPI network analyses can show the functional link between proteins and proteins, using string software (http://www.string-db.org) for PPI network analysis of differential genes. When building a protein interaction network, the settings are all set by default. Subsequently, Cytoscape software was applied to visualize and analyze the PPI network.

2.5. Module Analysis and Selection of Hub Genes. Molecular Complex Detection (MCODE) can find the interacting dense region in the PPI network, and the dense regions of interest can also be extracted and visualized. So we use MCODE to discover modules across the network. The hub genes were identified by using the plug-in cytoHubba of the Cytoscape software, including Maximal Clique Centrality (MCC), Density of Maximum Neighborhood Component (DMNC), and Maximum Neighborhood Component (MNC).

\section{Results}

3.1. Identification of DEGs. The results of standardizing the matrix file are shown in Figure 1. We identified a total of 25 DEGs in SCM samples compared with normal samples, including 10 upregulated genes and 15 downregulated genes, which were statistically significant (adjusted $p<0.05$, $\mid$ log fold change (FC) $\mid>1$ ) (Table 1). The cluster heatmap plot and volcano plot of the DEGs are shown in Figures 2 and 3.

3.2. GO Enrichment Analysis of DEGs. The GO analysis consists of biological processes (BP), cellular component (CC), and molecular function (MF) terms. The different genes with adjusted $p$ value $<0.05$ were obtained from GO functional enrichment. The GO enrichment analysis results reveal that the DEGs were mainly involved in the extracellular region, positive regulation of the multicellular organismal process, 


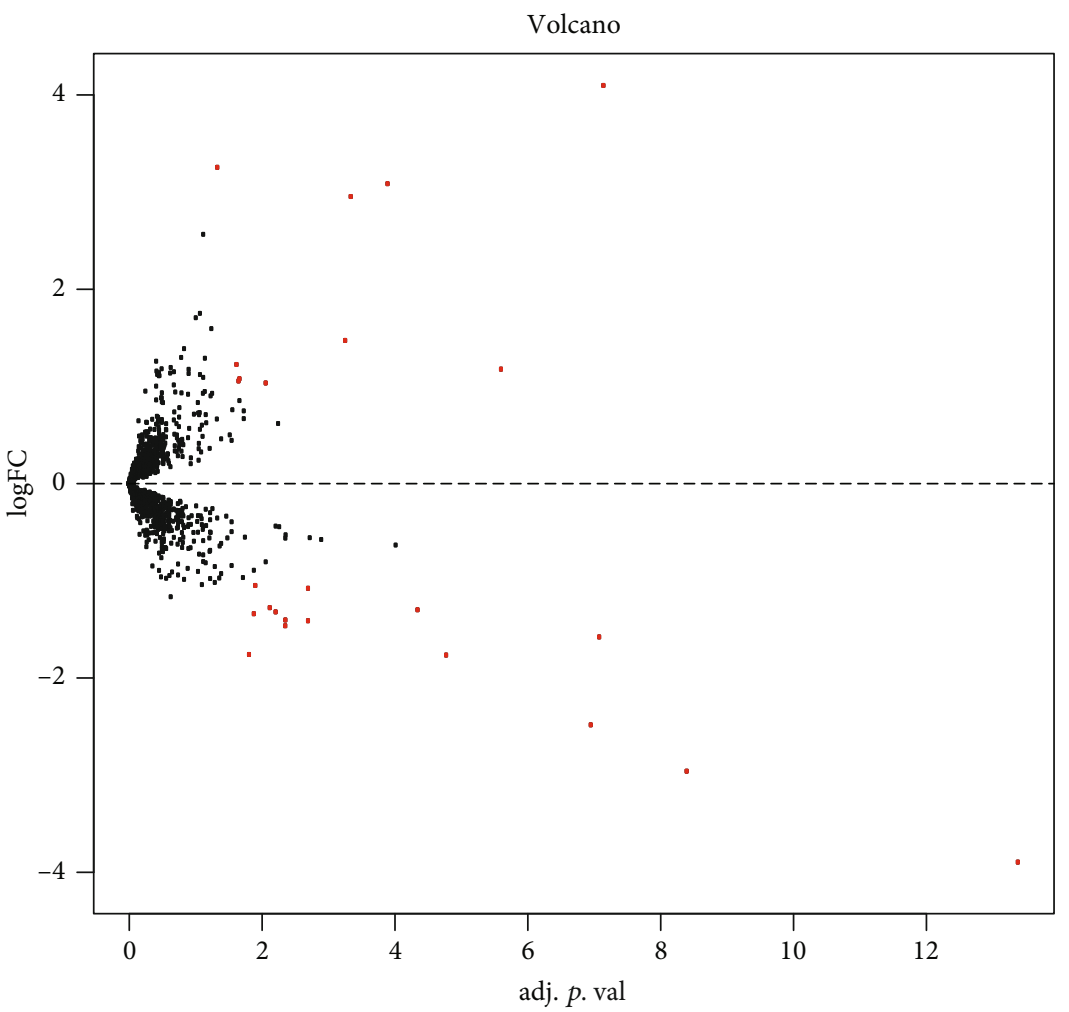

Figure 3: Differential expression of data between two sets of samples.

TABLE 2: GO enrichment analysis of differentially expressed genes.

\begin{tabular}{|c|c|c|c|}
\hline Term & Description & Count & $p$ value \\
\hline GO:0005576 & Extracellular region & 7 & $4.42 E-07$ \\
\hline GO:0051240 & Positive regulation of the multicellular organismal process & 5 & $5.94 E-07$ \\
\hline GO:0051234 & Establishment of localization & 7 & $6.12 E-07$ \\
\hline GO:0097708 & Intracellular vesicle & 5 & $8.56 E-07$ \\
\hline GO:0098802 & Plasma membrane signaling receptor complex & 3 & $1.31 E-06$ \\
\hline GO:1901576 & Organic substance biosynthetic process & 7 & $3.12 E-06$ \\
\hline GO:0030198 & Extracellular matrix organization & 3 & $6.13 E-06$ \\
\hline GO:0050794 & Regulation of cellular process & 8 & $6.88 E-06$ \\
\hline GO:0032102 & Negative regulation of response to external stimulus & 3 & $9.39 E-06$ \\
\hline GO:0008152 & Metabolic process & 8 & $1.10 E-05$ \\
\hline GO:0032270 & Positive regulation of cellular protein metabolic process & 4 & $1.32 E-05$ \\
\hline GO:0140096 & Catalytic activity, acting on a protein & 5 & $1.78 E-05$ \\
\hline GO:0031399 & Regulation of protein modification process & 4 & $1.89 E-05$ \\
\hline GO:0007229 & Integrin-mediated signaling pathway & 2 & $2.37 E-05$ \\
\hline GO:0050810 & Regulation of steroid biosynthetic process & 2 & $2.37 E-05$ \\
\hline GO:0120039 & Plasma membrane-bounded cell projection morphogenesis & 3 & $4.40 E-05$ \\
\hline GO:0001568 & Blood vessel development & 3 & $4.80 E-05$ \\
\hline GO:0030667 & Secretory granule membrane & 2 & $5.16 E-05$ \\
\hline GO:1990266 & Neutrophil migration & 2 & $5.84 E-05$ \\
\hline GO:0072562 & Blood microparticle & 2 & $7.34 E-05$ \\
\hline
\end{tabular}

Abbreviation: GO: Gene Ontology. 


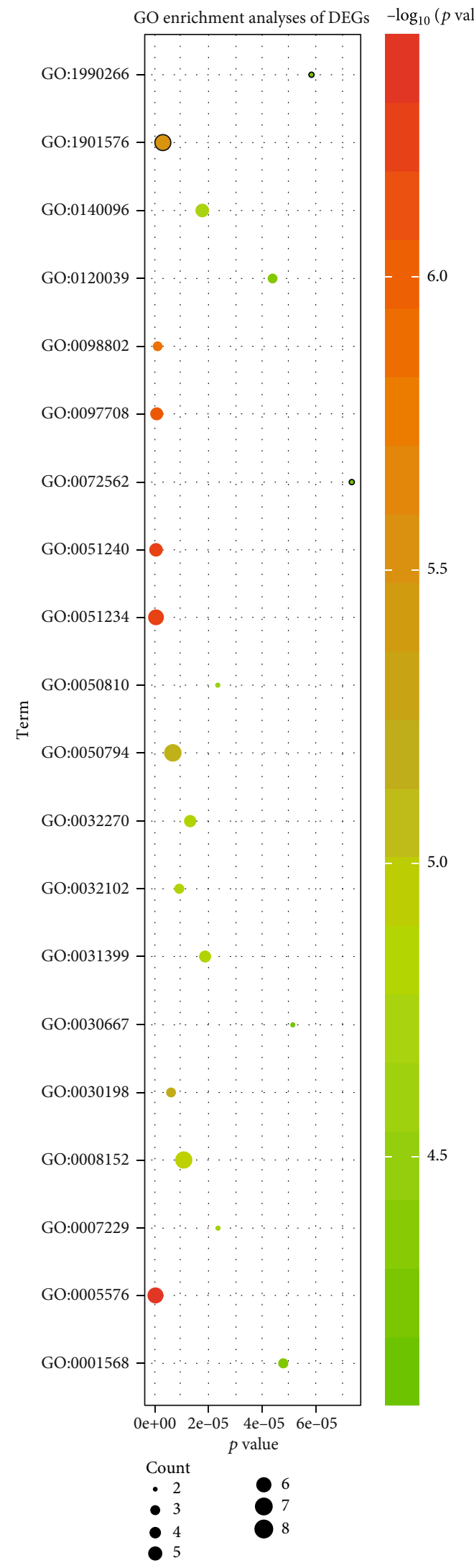

FIgUre 4: The DEGs significantly enriched GO (top 20). Abbreviation: DEGs, differentially expressed genes; GO, Gene Ontology. 
TABLE 3: KEGG pathway analysis of DEGs.

\begin{tabular}{|c|c|c|c|c|}
\hline Pathway & ID & Count & $p$ value & Genes \\
\hline ECM-receptor interaction & hsa04512 & 5 & $4.03 E-09$ & ITGA1|ITGB3|THBS2|ITGB1|ITGA2B \\
\hline Dilated cardiomyopathy (DCM) & hsa05414 & 5 & $6.84 E-09$ & ITGA1|ITGB3|PRKACA|ITGB1|ITGA2B \\
\hline Human papillomavirus infection & hsa05165 & 6 & $8.06 E-08$ & THBS2|ITGB1|ITGB3|PRKACA|ITGA1|ITGA2B \\
\hline Focal adhesion & hsa04510 & 5 & $2.31 E-07$ & ITGA1|ITGB3|THBS2|ITGB1|ITGA2B \\
\hline $\begin{array}{l}\text { Arrhythmogenic right ventricular cardiomyopathy } \\
\text { (ARVC) }\end{array}$ & hsa05412 & 4 & $2.55 E-07$ & ITGA1|ITGB3|ITGB1|ITGA2B \\
\hline Hypertrophic cardiomyopathy (HCM) & hsa05410 & 4 & $4.65 E-07$ & ITGA1|ITGB3|ITGB1|ITGA2B \\
\hline Platelet activation & hsa04611 & 4 & $1.60 E-06$ & ITGB3|PRKACA|ITGB1|ITGA2B \\
\hline Phagosome & hsa04145 & 4 & $3.52 E-06$ & ITGB3|THBS2|ITGB1|C3 \\
\hline PI3K-Akt signaling pathway & hsa04151 & 5 & $3.71 E-06$ & ITGA1|ITGB3|THBS2|ITGB1|ITGA2B \\
\hline Proteoglycans in cancer & hsa05205 & 4 & $1.08 E-05$ & ITGB3|PRKACA|ITGB1|PTPN6 \\
\hline Regulation of actin cytoskeleton & hsa04810 & 4 & $1.32 E-05$ & ITGA1|ITGB3|ITGB1|ITGA2B \\
\hline Leishmaniasis & hsa05140 & 3 & $1.89 E-05$ & ITGB1|PTPN6|C3 \\
\hline Complement and coagulation cascades & hsa04610 & 3 & $2.29 E-05$ & PLAT|A2M |C3 \\
\hline Hematopoietic cell lineage & hsa04640 & 3 & $4.15 E-05$ & ITGA1|ITGB3|ITGA2B \\
\hline Fluid shear stress and atherosclerosis & hsa05418 & 3 & 0.000117572 & ITGB3|PLAT|ITGA2B \\
\hline Cholesterol metabolism & hsa04979 & 2 & 0.00056172 & APOE $\mid$ APOB \\
\hline Thyroid hormone synthesis & hsa04918 & 2 & 0.001195438 & PRKACA $\mid$ ALB \\
\hline Pertussis & hsa05133 & 2 & 0.001258577 & ITGB1|C3 \\
\hline Rap1 signaling pathway & hsa04015 & 3 & 0.00038722786 & ITGB3|ITGB1|ITGA2B \\
\hline ECM-receptor interaction & hsa04512 & 5 & $4.03 E-09$ & ITGA1|ITGB3|THBS2|ITGB1|ITGA2B \\
\hline
\end{tabular}

Abbreviation: KEGG: Kyoto Encyclopedia of Genes and Genomes; DEGs: differentially expressed genes.

establishment of localization, and intracellular vesicle (Table 2, Figure 4).

3.3. KEGG Pathway Analysis of DEGs. The KEGG pathways of the DEGs were analyzed using DAVID and KOBAS. The top 20 of the KEGG pathways is shown in Table 3 and Figure 5, the DEGs chiefly enriched in ECM-receptor interaction, dilated cardiomyopathy (DCM), human papillomavirus infection, and focal adhesion.

3.4. Establishing the PPI Network, Conducting Module Analysis, and Selection of Hub Genes. Use the string online tool to create a PPI network to gain a better understanding of the biological properties of DEGs. There were 24 nodes and 51 edges in this network, as shown in Figure 6. Subsequently, Cytoscape is applied to confirm a vital module throughout the network; the most significant modules were selected, as shown in Figure 7. Nine key genes were identified, including SAA1, C3, CRP, ALB, APOE, APOB, MFGE8, GAPDH, and PLAT, finally utilizing the cytoHubba plug-in to determine APOE, MFGE8, ALB, APOB, SAA1, A2M, and $\mathrm{C} 3$ as the hub gene, as shown in Figure 8 and Table 4.

\section{Discussion}

In this study, we performed an integrated analysis of gene expression profiles from serum samples without/with SCM aiming to identify the DEGs, related key signaling pathways, and hub genes for the disease. A total of 25 DEGs, including 10 upregulated and 15 downregulated genes, were identified from the GSE95368 database. The GO enrichment analysis showed that these differential genes associated with SCM were mainly enriched in the extracellular region, positive regulation of the multicellular organismal process, establishment of localization, and intracellular vesicle. From the KEGG pathway enrichment analysis, we identified that these DEGs were mainly enriched in the pathway of the ECMreceptor interaction and dilated cardiomyopathy (DCM). Through the construction and module analysis of the PPI network, we identified 9 key genes, including SAA1, C3, CRP, ALB, APOE, APOB, MFGE8, GAPDH, and PLAT. Finally, APOE, MFGE8, ALB, APOB, SAA1, A2M, and C3 are regarded as hub genes for the development of SCM.

MFGE8, a secreted glycoprotein, is associated with a variety of pathophysiological processes, including anti-inflammatory [11], antifibrosis [12], antiatherosclerosis [13], and inhibition of cardiac hypertrophy [14]. Recent studies have shown that MFGE8 is a part of the arterial inflammatory signaling network that promotes endothelial cell apoptosis [15]. MFGE8 is an inflammatory mediator that coordinates multiple cellular interactions and is involved in the pathogenesis of various diseases. In a study investigating postischemic injury, MFGE8 may alleviate postischemic injury by integrin beta3-dependent inhibition of inflammatory bodies [15]. Cardiomyocyte ischemia plays a critical role in the development of SCM, so we propose that MFGE8 may play an important role in SCM by participating in the process of myocardial ischemia.

C3 is the most abundant complement component in serum, mainly macrophage and liver synthesis, and plays 


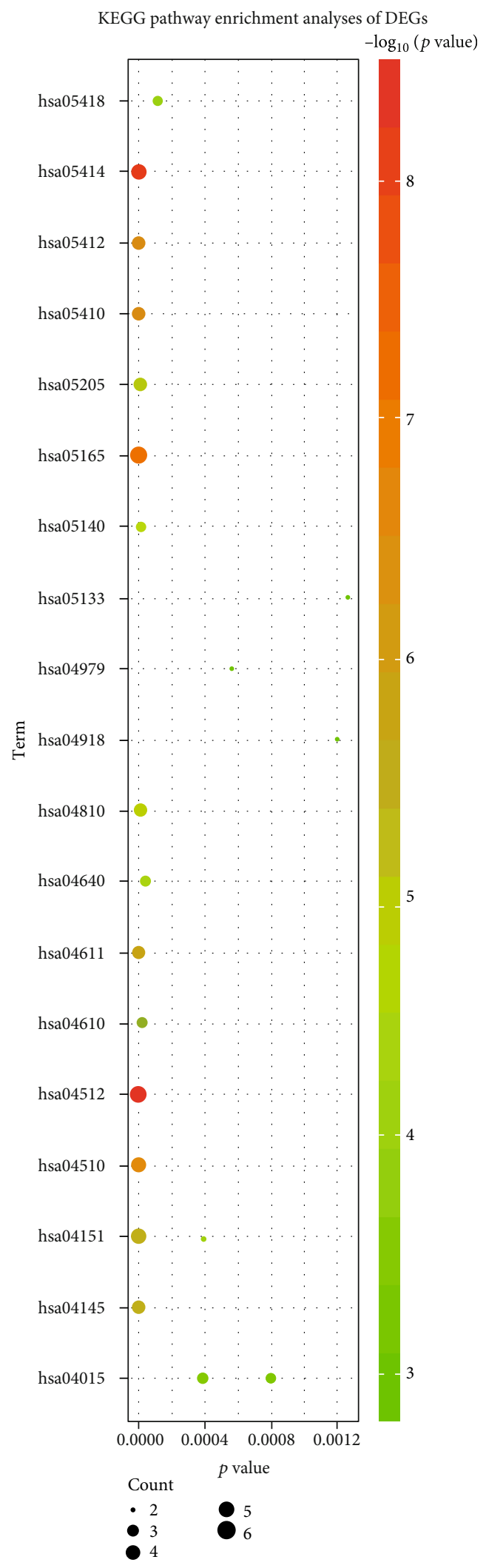

FIGURE 5: KEGG pathway analysis of DEGs. Abbreviation: KEGG: Kyoto Encyclopedia of Genes and Genomes; DEGs: differentially expressed genes. 


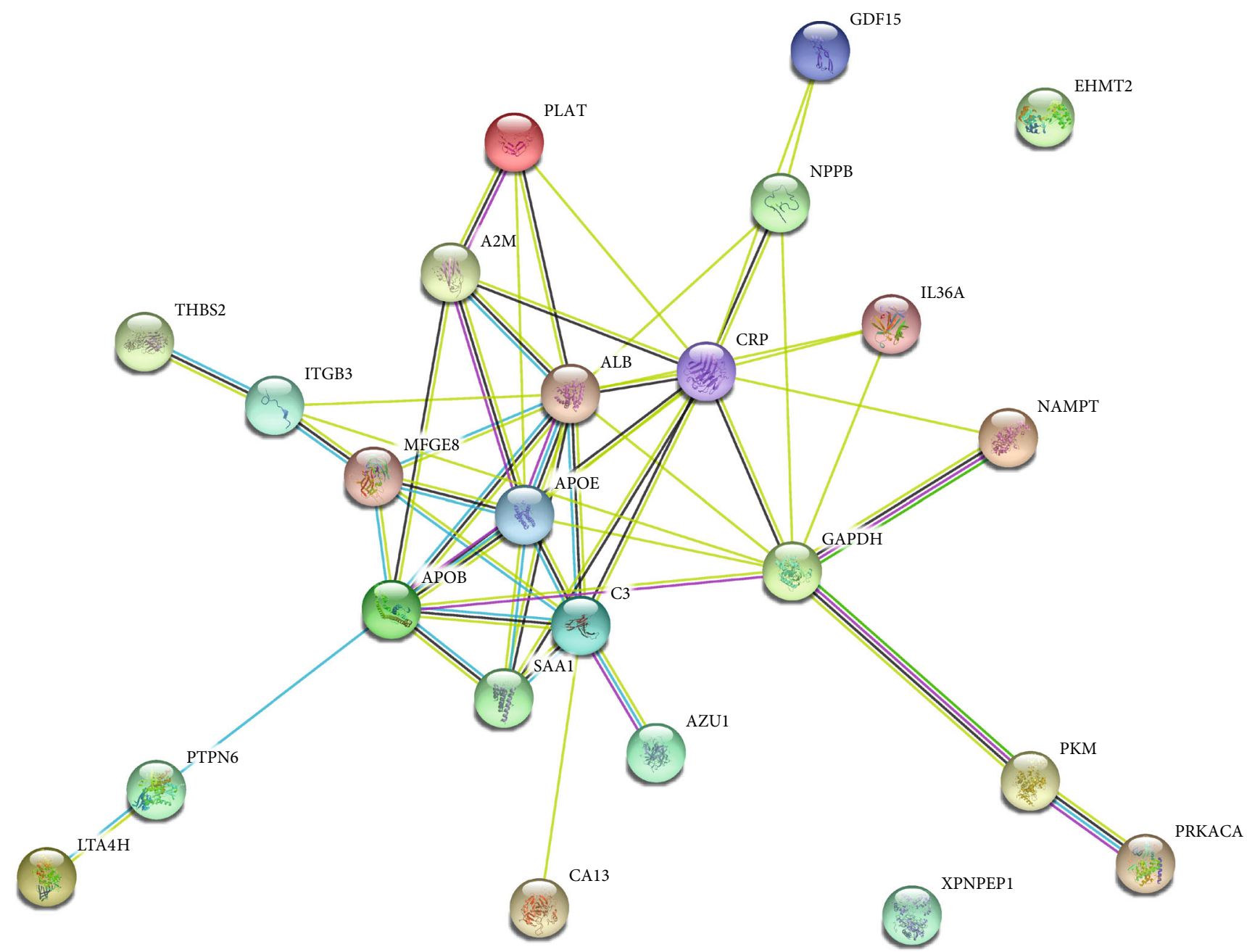

Figure 6: Results of PPI network analysis of DEGs. Abbreviation: PPI: protein-protein interaction; DEGs: differentially expressed genes.

an important role in complementing the classical activation pathway and bypass activation pathway [16]. C3 increases are common in the early stages of some acute inflammation or infectious diseases [17]. Although the specific mechanism of SCM is unclear, most patients are subjected to stress status. C3 is one of many neurohumoral factors released as a result of a series of adaptive responses in the body under stress [18]. So C3 may be involved in the pathological process of SCM through this pathway. SAA promotes chemotaxis of monocytes and neutrophils and plays a key role in various functions such as lipoprotein metabolism, cholesterol transport, and host defense [19]. Previous studies have shown that SAA1 plasma levels have increased dramatically in response to tissue damage, infection, and various emergencies [20, 21]. So SAA1 is also involved in the pathogenesis of SCM, and its mechanism is similar to that of C3.

Alpha-2-macroglobulin (A2M) is a broad-spectrum protease-binding protein of the vertebrate innate immune system that prevents pathogen invasion [22, 23]. In our study, both A2M and C3 were enriched in the complement and coagulation cascade pathway, suggesting an important role in coagulation. A recent study suggests that
SCM is a prethrombotic state [24], which is consistent with our findings, so we speculate that A2M and $\mathrm{C} 3$ may be involved in the SCM pathological process through this pathway.

Serum albumin (ALB) is synthesized in the liver and is the most abundant protein in vertebrate plasma. Its main function is to maintain plasma colloid osmotic pressure and participate in the transport of various substances $[25,26]$. We found that ALB is mainly associated with the synthesis of thyroid hormones, which increase in response to human mood swings and emergencies [27]. So thyroid hormone is involved in the process of SCM, and therefore, ALB is also likely to be involved in the pathogenesis of SCM. In addition, several studies have shown that serum ALB has a predictive value for a variety of thrombotic diseases [28, 29]. As mentioned above, SCM is also a prethrombotic state, so we guess that serum ALB also seems to have some predictive effect on SCM.

Apolipoprotein is a protein that can bind and transport blood lipids to tissues for metabolism and utilization [30]. A large number of studies have found that apolipoprotein gene mutation can affect blood lipid metabolism and 


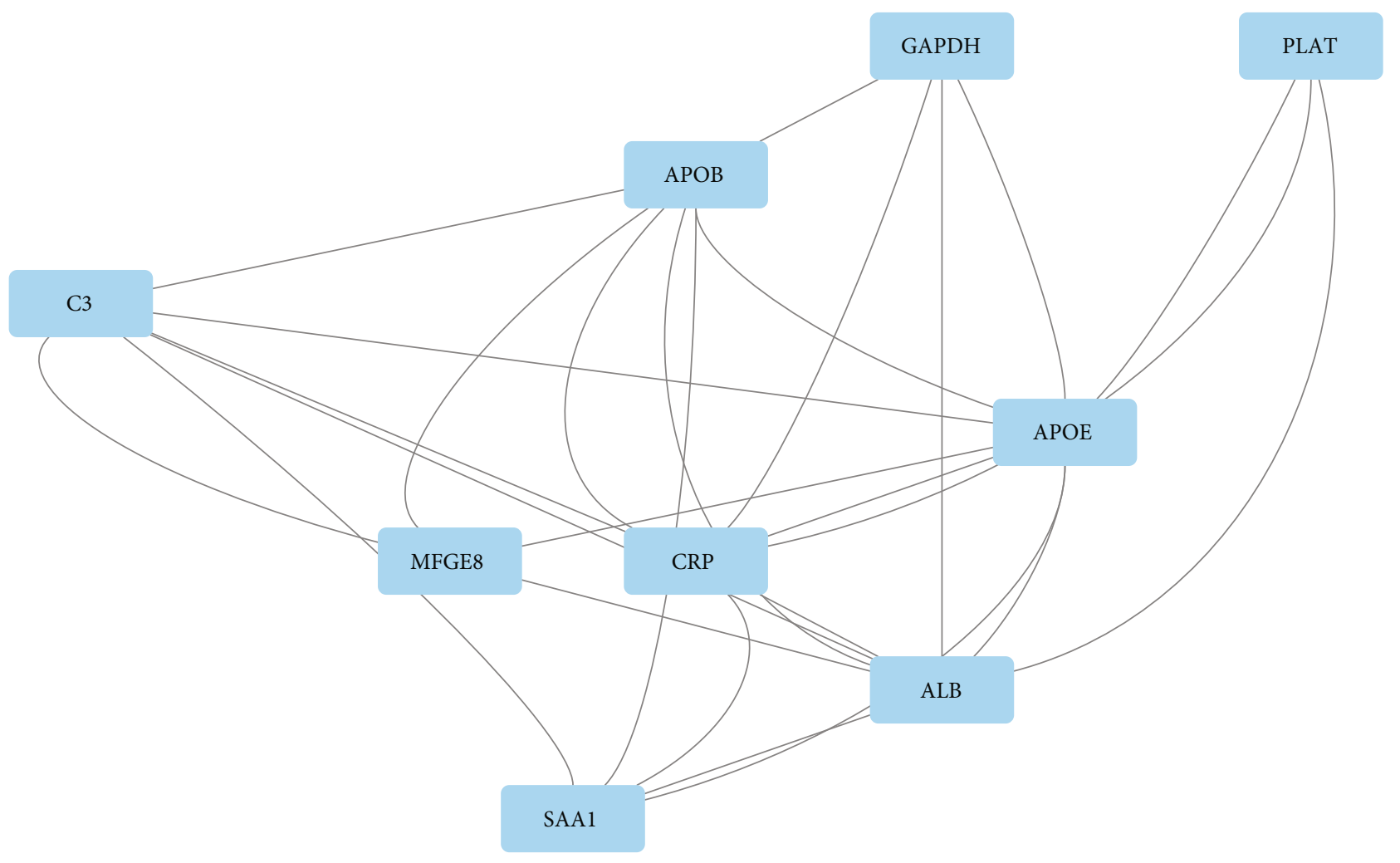

FIgURE 7: PPI network of module. Abbreviation: PPI: protein-protein interaction.

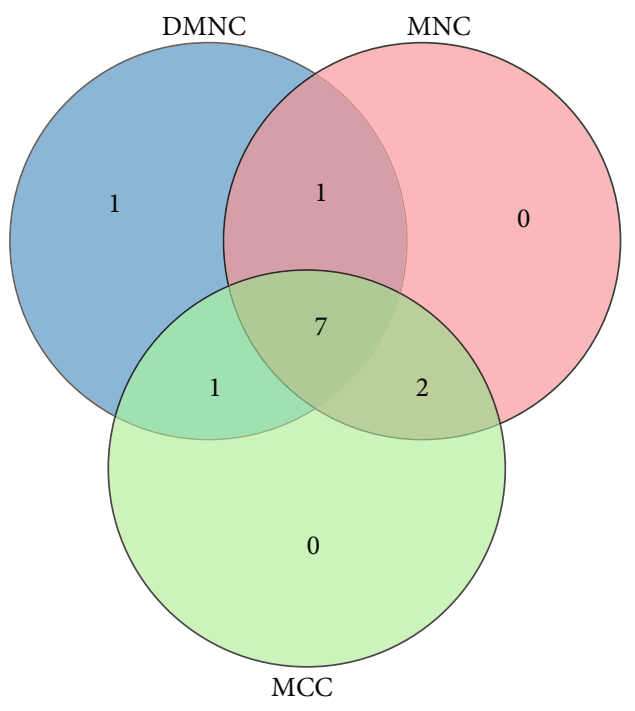

Figure 8: Venn diagram of common hub genes based on three methods. Abbreviation: MCC: Maximal Clique Centrality; DMNC: Density of Maximum Neighborhood Component; MNC: Maximum Neighborhood Component.

utilization, thus growing up to be a risk factor for cardiovascular and cerebrovascular diseases, diabetes, and other diseases [31,32]. APOE and APOB are members of the apolipoprotein family, and so far, no studies have shown a direct relationship between apolipoprotein and SCM. Previous studies have shown that diabetes is a risk factor for SCM,
TABLE 4: Hub genes based on cytoHubba.

\begin{tabular}{lccc}
\hline \multirow{2}{*}{ Projects } & \multicolumn{3}{c}{ Methods in cytoHubba } \\
& MCC & MNC & DMNC \\
\hline \multirow{4}{*}{ ALB } & ALB & SAA1 \\
& APOE & CRP & C3 \\
& CRP & APOE & A2M \\
& APOB & APOB & PLAT \\
& C3 & GAPDH & APOB \\
& SAA1 & C3 & APOE \\
& A2M & A2M & IL36A \\
& GAPDH & MFGE8 & MFGE8 \\
& MFGE8 & SAA1 & ALB \\
& PLAT & NPPB & NPPB \\
\hline
\end{tabular}

Bold gene symbols were the overlap hub gene. Abbreviation: MCC: Maximal Clique Centrality; DMNC: Density of Maximum Neighborhood Component; MNC: Maximum Neighborhood Component.

and hyperlipidemia is a risk factor for diabetes [33, 34], so it can be speculated that hyperlipidemia is a potential risk factor for SCM, so compared with the normal population, APOE and APOB genes are upregulated in SCM patients.

In this experiment, we analyzed gene chips to obtain SCM possible key genes and related pathway information. However, due to the defects of the study itself, the conclusion needs basic and clinical experimental verification. Most regrettably, due to the limited experimental conditions, the conclusions drawn in this paper cannot be further 
investigated. But we hope to be able to provide new ideas for SCM diagnosis based on this study and expect other scientific researchers to further explore this.

\section{Conclusions}

In summary, our study provides an integrated bioinformatics analysis of DEGs of SCM. In the present study, we identified some key genes and pathways. However, the key genes and signaling pathways related to SCM derived from this study still need further experimental verification due to the defects of analytical methods and sample size.

\section{Data Availability}

The data used to support the findings of this study are included within the supplementary information file.

\section{Conflicts of Interest}

The authors declare that they have no conflicts of interest.

\section{Supplementary Materials}

GSE95368 datasets were downloaded from GEO (http://www .ncbi.nlm.nih.gov/geo/). (Supplementary Materials)

\section{References}

[1] R. T. Hurst, A. Prasad, J. W. Askew, P. P. Sengupta, and A. J. Tajik, "Takotsubo cardiomyopathy: a unique cardiomyopathy with variable ventricular morphology," JACC: Cardiovascular Imaging, vol. 3, no. 6, pp. 641-649, 2010.

[2] M. Lee, "Time course of functional recovery in takotsubo (stress) cardiomyopathy: a serial speckle tracking echocardiography and electrocardiography study," Journal of Cardiovascular Imaging, vol. 28, no. 1, pp. 50-60, 2020.

[3] M. W. Roach and P. Currie, “'Tax-otsubo': stress cardiomyopathy following an encounter with the Inland Revenue," BMJ case reports, vol. 13, no. 1, article e232225, 2020.

[4] N. Câmara, E. Sierra, C. Fernández-Maldonado et al., "Stress cardiomyopathy in stranded cetaceans: a histological, histochemical and immunohistochemical study," The Veterinary Record, vol. 185, no. 22, p. 694, 2019.

[5] A. R. Lyon, E. Bossone, B. Schneider et al., "Current state of knowledge on takotsubo syndrome: a position statement from the Taskforce on Takotsubo Syndrome of the Heart Failure Association of the European Society of Cardiology," European Journal of Heart Failure, vol. 18, no. 1, pp. 8-27, 2016.

[6] S. W. Sharkey and B. J. Maron, "Epidemiology and clinical profile of takotsubo cardiomyopathy," Circulation Journal, vol. 78, no. 9, pp. 2119-2128, 2014.

[7] G. Novo, S. Giambanco, V. Bonomo et al., "Troponin I/ejection fraction ratio: a new index to differentiate takotsubo cardiomyopathy from myocardial infarction," International Journal of Cardiology, vol. 180, pp. 255-257, 2015.

[8] H. Zhang, Y. Sun, X. Liu et al., "Speckle tracking echocardiography in early detection of myocardial injury in a rat model with stress cardiomyopathy," Medical Ultrasonography, vol. 21, no. 4, pp. 441-448, 2019.
[9] A. Briasoulis, K. Marinescu, M. Mocanu et al., "Comparison of left ventricular contractile abnormalities in stress-induced cardiomyopathy versus obstructive coronary artery disease using two-dimensional strain imaging," Echocardiography, vol. 33, no. 6, pp. 863-870, 2016.

[10] H. Medina de Chazal, M. G. del Buono, L. Keyser-Marcus et al., "Stress Cardiomyopathy Diagnosis and Treatment:," Journal of the American College of Cardiology, vol. 72, no. 16, pp. 1955-1971, 2018.

[11] J. Viola, P. Lemnitzer, N. Paulin et al., "Deletion of MFGE8 inhibits neointima formation upon arterial damage," Thrombosis and Haemostasis, vol. 118, no. 7, pp. 1340-1342, 2018.

[12] Y. J. Jang, S. Y. An, and J.-H. Kim, "Identification of MFGE8 in mesenchymal stem cell secretome as an anti-fibrotic factor in liver fibrosis," BMB Reports, vol. 50, no. 2, pp. 58-59, 2017.

[13] H. Zhao, H. Zhang, and X. Qin, “Age-related differences in serum MFG-E8, TGF- $\beta 1$ and correlation to the severity of atherosclerosis determined by ultrasound," Molecular Medicine Reports, vol. 16, no. 6, pp. 9741-9748, 2017.

[14] K. Q. Deng, J. Li, Z. G. She et al., "Restoration of circulating MFGE8 (milk fat globule-EGF factor 8) attenuates cardiac hypertrophy through inhibition of Akt pathway," Hypertension, vol. 70, no. 4, pp. 770-779, 2017.

[15] N. Deroide, X. Li, D. Lerouet et al., "MFGE8 inhibits inflammasome-induced IL- $1 \beta$ production and limits postischemic cerebral injury," The Journal of Clinical Investigation, vol. 123, no. 3, pp. 1176-1181, 2013.

[16] S. Huang, J. Tian, C. Liu et al., "Elevated C-reactive protein and complement C3 levels are associated with preterm birth: a nested case-control study in Chinese women," BMC Pregnancy and Childbirth, vol. 20, no. 1, p. 131, 2020.

[17] P. F. Zipfel, T. Wiech, E. D. Stea, and C. Skerka, "CFHRGene variations provide insights in the pathogenesis of the kidney diseases atypical hemolytic uremic syndrome and C3 glomerulopathy," Journal of the American Society of Nephrology, vol. 31, no. 2, pp. 241-256, 2020.

[18] Y. Zhang, Z. Zeng, G. Xiao, W. Zhang, W. Lin, and J. Deng, “A randomized trial to evaluate a modified tracheal catheter with upper and lower balloons for anesthetic administration: effect on the cardiovascular, stress response, and comfort in patients undergoing laparoscopic cholecystectomy," BMC Anesthesiology, vol. 19, no. 1, p. 211, 2019.

[19] B. Y. Wang, J. Y. Hang, Y. Zhong, and S. J. Tan, “Association of genetic polymorphisms of SAA1 (rs12218) with myocardial infarction in a Chinese population," Genetics and Molecular Research, vol. 13, no. 2, pp. 3693-3696, 2014.

[20] C. Jumeau, F. Awad, E. Assrawi et al., "Expression of SAA1, SAA2 and SAA4 genes in human primary monocytes and monocyte-derived macrophages," PLoS One, vol. 14, no. 5, article e0217005, 2019.

[21] L. Jin, T. Syrovets, J. S. Scheller, X. Zhang, and T. Simmet, "Comparative study on hyaluronic acid binding to murine SAA1.1 and SAA2.2," ACS Omega, vol. 4, no. 8, pp. 13388-13399, 2019.

[22] V. Mohindra, T. Dangi, L. M. Chowdhury, and J. K. Jena, “Tissue specific alpha-2-macroglobulin (A2M) splice isoform diversity in hilsa shad, Tenualosa ilisha (Hamilton, 1822)," PLoS One, vol. 14, no. 7, article e0216144, 2019.

[23] X. Chen, Z. H. Xie, Y. X. Lv et al., "A proteomics analysis reveals that A2M might be regulated by STAT3 in persistent allergic rhinitis," Clinical and Experimental Allergy, vol. 46, no. 6, pp. 813-824, 2016. 
[24] T. P. Fitzgibbons, Y. J. K. Edwards, P. Shaw et al., “Activation of inflammatory and pro-thrombotic pathways in acute stress cardiomyopathy," Frontiers in Cardiovascular Medicine, vol. 4, p. 49, 2017.

[25] J. M. Shi, Z. Yang, F. Q. Li, and G. J. Wang, "Preliminary study of human serum albumin level in early warning onset of preeclampsia," Zhonghua Fu Chan Ke Za Zhi, vol. 55, no. 1, pp. 29-35, 2020.

[26] H. Luo, Y. Fu, C. You, C. Liu, and F. Xu, "Comparison of admission serum albumin and hemoglobin as predictors of outcome in children with moderate to severe traumatic brain injury: a retrospective study," Medicine, vol. 98, no. 51, article e18562, 2019.

[27] B. McKinnon, H. Li, K. Richard, and R. Mortimer, "Synthesis of thyroid hormone binding proteins transthyretin and albumin by human trophoblast," The Journal of Clinical Endocrinology and Metabolism, vol. 90, no. 12, pp. 6714-6720, 2005.

[28] T. Dziedzic, A. Slowik, and A. Szczudlik, "Serum albumin level as a predictor of ischemic stroke outcome," Stroke, vol. 35, no. 6, pp. e156-e158, 2004.

[29] V. Oduncu, A. Erkol, C. Y. Karabay et al., "The prognostic value of serum albumin levels on admission in patients with acute ST-segment elevation myocardial infarction undergoing a primary percutaneous coronary intervention," Coronary Artery Disease, vol. 24, no. 2, pp. 88-94, 2013.

[30] J. W. Meeusen, "Apolipoprotein B or low-density lipoprotein cholesterol: is it time for a twenty-first-century lipid marker?," Clinical Chemistry, vol. 64, no. 6, pp. 984-985, 2018.

[31] L. Lu, Y. Qin, C. Chen et al., "The atheroprotective roles of heart-protecting musk pills against atherosclerosis development in apolipoprotein E-deficient mice," Annals of translational medicine, vol. 7, no. 23, p. 714, 2019.

[32] T. Onoue, M. Goto, E. Wada et al., "Dipeptidyl peptidase-4 inhibitor anagliptin reduces fasting apolipoprotein B-48 levels in patients with type 2 diabetes: a randomized controlled trial," PLoS One, vol. 15, no. 1, article e0228004, 2020.

[33] T. Stiermaier, F. Santoro, I. el-Battrawy et al., "Prevalence and prognostic impact of diabetes in takotsubo syndrome: insights from the international, multicenter GEIST Registry," Diabetes Care, vol. 41, no. 5, pp. 1084-1088, 2018.

[34] J. E. Madias, "Low prevalence of diabetes mellitus in patients with takotsubo syndrome: a plausible "protective" effect with pathophysiologic connotations," European Heart Journal Acute Cardiovascular Care, vol. 5, no. 2, pp. 164-170, 2015. 